\title{
Síntomas clínicos, subclínicos y necesidades de atención psicológica en estudiantes universitarios con bajo rendimiento
}

Mayorga-Lascano, Marlon; Moreta-Herrera, Rodrigo

Síntomas clínicos, subclínicos y necesidades de atención psicológica en estudiantes universitarios con bajo rendimiento

Revista Educación, vol. 43, núm. 2, 2019

Universidad de Costa Rica, Costa Rica

Disponible en: http://www.redalyc.org/articulo.oa?id=44058158036

DOI: https://doi.org/10.15517/revedu.v43i2.32239

Esta obra está bajo una Licencia Creative Commons Atribución-NoComercial-SinDerivar 3.0 Internacional. 


\title{
Síntomas clínicos, subclínicos y necesidades de atención psicológica en estudiantes universitarios con bajo rendimiento
}

\author{
Psychological Care Needs for Underperforming College Students with Clinical and Subclinical Symptoms \\ Marlon Mayorga-Lascano \\ Escuela de Psicología de la Pontificia Universidad Católica Redalyc: http://www.redalyc.org/articulo.oa?id=44058158036 \\ del Ecuador, Ecuador \\ pmayorga@pucesa.edu.ec
}

(D) http://orcid.org/0000-0002-2515-4159

Rodrigo Moreta-Herrera

Escuela de Psicología de la Pontificia Universidad Católica

del Ecuador, Ecuador

rmoreta@pucesa.edu.ec

(iD http://orcid.org/0000-0003-0134-5927

Recepción: 08 Junio 2018
Aprobación: 10 Junio 2019

\section{Resumen:}

El objetivo de la investigación es establecer la presencia de sintomatología psicológica clínica y subclínica, niveles de riesgo y necesidad de atención psicológica en el estudiantado de la Escuela de Psicología de la PUCESA que presenta bajo rendimiento académico. La investigación posee un diseño no experimental, de tipo cuantitativo, de alcance descriptivo y comparativo y de corte transversal. Participaron 92 estudiantes de las carreras de Psicología Clínica y Organizacional, divididos en 77,2\% (mujeres) y 22,8\% (hombres); evaluados con el Inventario de los 90 Síntomas revisado (SCL-90-R) de Derogatis, Rickels y Rock (1976). El 65,1\% de los examinados presentaron bajo rendimiento académico (condicionamiento académico), mientras el 34,4\% estuvo constituido por estudiantes no condicionados como grupo de comparación. Los resultados revelan que el estudiantado con primer condicionamiento muestra mayor prevalencia en ideas y comportamientos obsesivo-compulsivos, sintomatología depresiva, somatizaciones y en menor medida ansiedad, ideación paranoide y hostilidad; en el estudiantado con segundo condicionamiento los síntomas obsesivo-compulsivos disminuyen, la ansiedad casi desaparece, mientras que la depresión y la hostilidad aumentan, aunque sin alcanzar niveles clínicos; no existen diferencias significativas con el grupo control. Un 19,1\% de los examinados se encuentra en riesgo debido a su sintomatología, mientras que un 3,5\% presenta necesidades de atención psicológica.

Palabras ClaVe: síntomas psicológicos, niveles de riesgo, atención psicológica, estudiantes universitarios.

\section{ABstract:}

This study reports on clinical and subclinical psychological symptoms, risk levels and psychological care requirements identified in psychology majors with low academic achievement at the Catholic Pontifical University of Ecuador (PUCESA) This study is used a non-experimental design which is quantitative in nature, descriptive, comparative and cross-sectional. A sample group of 92 students were selected specifically among students majoring in Clinical and Organizational Psychology. The Revised Symptom Checklist - 90-R (SCL 90-R) by Derogatis, Rickels and Rock (1976) was used to assess the sample group of $77.2 \%$ women and $22.8 \%$ men. Approximately $65.1 \%$ of the participants displayed low academic performance (academic conditioned) while 34.4 made up the control group of students (unconditioned) for a head-to-head comparison. Based on the results, the first academic conditioned group had a higher prevalence of obsessive-compulsive ideation and behaviors with symptoms of depression, somatization, anxiety, paranoid ideation and hostility, though to a lesser extent. While students in the second non-condition group had fewer obsessive-compulsive symptoms and low anxiety, they had higher rates of depression and hostility which did not, however, attain clinical levels. Although no significant differences were found with the control group, $19.1 \%$ were deemed to be at risk for developing psychological disorders due to their symptoms, while 3.5\% required psychological counseling.

KeYwORDS: Psychological Symptoms, Risk Levels, Psychological Attention, University Students. 


\section{INTRODUCCIÓN}

El modelo teórico de la presente investigación se adscribe a un paradigma objetivista, el cual toma como objeto de estudio los procesos psicológicos que incluyen conductas, rasgos, cogniciones, motivaciones, es decir, todo lo que pueda ser definido operativamente y medido con instrumentos fiables. Paralelamente, el modelo utilizado es factorial biológico, basado en la influencia ejercida por los factores fisiológicos y constitucionales a la hora de caracterizar los rasgos de la estructura de la personalidad (Cano, García, Rodríguez y Antuña, 2005).

El rendimiento académico del estudiantado representa un indicador consensuado de calidad educativa (Marshall y Correa, 2003); las notas obtenidas certifican de manera precisa los niveles alcanzados, y se asume que reflejan logros académicos en los diferentes componentes del aprendizaje, los cuales incluyen aspectos personales, académicos y sociales (Rodríguez, Fita y Torrado, 2004). El estudio del desempeño académico y el bajo rendimiento educativo del estudiantado, revela la existencia de una asociación entre estado de ánimo y rendimiento (Gómez y Rodríguez, 1997). Dado que los universitarios son el segmento poblacional el cual muestra mayores problemas de salud mental (Moreira y Telzer, 2015), el presente trabajo busca establecer la presencia de síntomas psicológicos en el alumnado que presenta bajo rendimiento académico, así como el riesgo y la necesidad de atención asociada a dicha condición. Pues, se hipotetiza la prevalencia de sintomatología clínica y subclínica en este segmento poblacional.

Las exigencias del actual sistema de educación superior, acrecientan la sobrecarga académica y los consecuentes niveles de ansiedad ante la evaluación, los cuales se manifiestan a través de tres canales: fisiológico, cognitivo y conductual (Núñez, 2001). Las estrategias de afrontamiento que el estudiantado emplea ante la problemática, tiende a ser inadecuada, por lo que termina por convertirse en un factor que les vuelve más vulnerable a las situaciones y experiencias académicas potencialmente estresantes y a presentar bajo rendimiento académico (Cabanach, Rodríguez, Valle Piñeiro y Millán, 2007; Tran et ál., 2017); lo que determinará el desarrollo o la instalación de estrés académico, malestar psicoemocional y sintomatología psicológica consecuente.

Debido a ello, en el estudiantado que presenta bajo rendimiento académico, se evidencian altos niveles de estrés, los cuales tienden a ser mayores en las mujeres que en los hombres (Del Toro, Gorguet, Pérez y Gorguet, 2011). Es esperado que en la población universitaria los niveles adecuados de bienestar individual y el social predigan una salud mental positiva (Moreta et ál., 2018); sin embargo, los estudios revelan el aparecimiento de sintomatología ansiosa y depresiva en este tipo de población (Osada, Rojas, Rosales y Vega, 2010), la cual puede alcanzar una intensidad, duración y frecuencia de nivel clínico (Masi, Sbrana, Poli, Tomaiuolo, Favilla y Marcheschi, 1998; Campo y Cassiani, 2008).

Debe anotarse que factores como la familia y el apoyo social promueven la capacidad de los sujetos a resistir y sobreponerse a situaciones adversas, en este caso académicas; mientras que la acción de fortalezas individuales y colectivas aseguran el desarrollo sano y promueven la transformación positiva del entorno social de la institución (Melillo y Suárez, 2008). Además, la movilización de más factores de resiliencia está asociada al mejoramiento del rendimiento académico (Villalta, 2010).

\section{ANTECEDENTES}

La presente investigación parte de la existencia de un 27,9\% del estudiantado de la Escuela de Psicología de la PUCESA que presenta un rendimiento inferior al índice promedial (35/50 puntos), el cual se obtiene al dividir la sumatoria de las calificaciones alcanzadas por un estudiante durante un semestre, para el total de créditos de dicho semestre; las notas inferiores a 35/50 puntos constituyen un condicionamiento académico; entendiéndose por condicionamiento académico a una calificación inferior a la fijada por cada unidad académica y cuya presencia reiterada constituye un impedimento para continuar en la carrera y quedar 
excluido de ella (Pontificia Universidad Católica del Ecuador Sede Ambato, 2011). El reglamento de la PUCESA admite hasta dos condicionamientos consecutivos; si posterior a eso, el promedio de 35/50 no es alcanzado, el estudiante queda fuera de la carrera de manera permanente y definitiva. Ello ha determinado que un $5 \%$ de la población estudiantil haya sido separada, tras presentar más de dos condicionamientos consecutivos (Secretaría Escuela de Psicología PUCESA, 2014).

A partir de lo señalado, la ocurrencia del condicionamiento académico, coloca al estudiante en una situación de vulnerabilidad y riesgo de desarrollar síntomas psicológicos. La problemática descrita, produce consecuencias individuales como la deserción académica y la consecuente limitación para acceder a trabajos bien remunerados, así como una menor participación social y política. Debido a ello, la necesidad de combatir el bajo rendimiento es una tarea relevante para los sistemas educativos, pues representa una mejoría sustancial de la sociedad misma (Backhoff, 2016).

En Ecuador, la investigación de Mayorga (2013) evidencia que estudiantes de universidad con problemas cognitivos, emocionales y conductuales presentan mayores problemas de rendimiento académico que el resto de sus pares. También que estas dificultades no se desprenden de trastornos de personalidad y por tanto se infiere que su etiología reside en la presencia de sintomatología clínica o más precisamente subclínica, por tratarse de una población que en apariencia se encuentra dentro de límites de normalidad (Mayorga, 2014).

\section{MARCO TEÓRICO}

La sintomatología psicológica subclínica se refiere a alteraciones que no se expresan con signos y síntomas detectables; pero se revelan a partir de los efectos que generan en el desempeño y funcionalidad general del sujeto (Blanco, 2005). Aunque la sintomatología subclínica no cumpla con los criterios necesarios para poder establecer un diagnóstico psicopatológico (cantidad, duración y frecuencia), también se puede evidenciar a partir de la aplicación de pruebas psicométricas, donde la prevalencia encontrada, a menudo supera las expresiones clínicas o psicopatológicas habituales (Bramajo y López, 2013).

Trastornos de interiorización como la depresión y la ansiedad poseen manifestaciones subclínicas. La sintomatología subclínica de la depresión tiende a aparecer como una respuesta inadecuada ante las crisis evolutivas y eventos que naturalmente se dan en la vida de todas las personas (Paolini, 2005); mientras que las variantes subclínicas de la ansiedad se expresan en preocupaciones entre un $25 \%$ a $30 \%$ más prevalentes que los trastornos ansiosos clínicamente detectados (entre el 6 y el 7\%) (Jaude, 2002).

Trastornos de exteriorización, como los alimenticios y los de consumo de sustancias también poseen manifestaciones subclínicas, estas ostentan una alta prevalencia y pueden activarse bajo condiciones desfavorables. Así, la anorexia y la bulimia presenta una prevalencia subclínica 10 veces superior a la de los casos clínicos encontrados (Behar, González, Ariza, y Aguirre, 2008); mientras que la ingestión alcohólica exhibe un periodo subclínico detectable en las pruebas psicométricas, muy superior al de la sintomatología clínica habitual (Bramajo y López, 2013).

La sintomatología clínica hace referencia a la percepción subjetiva del sujeto al respecto de su propia salud, la cual se concretiza y vuelve evidente a través del comportamiento que se desprende de ella misma (Jiménez, 2012). Los límites que separan la conducta normal de la anormal no están completamente establecidos, esto no implica que no sean factibles de definirse, pues más allá de las divergencias existentes, la conducta anormal o patológica suele cumplir los siguientes criterios: intensidad, duración y frecuencia especificada, ser percibida como irracional para el sujeto que la despliega, ser perjudicial para quien la utiliza y para los otros, e impedir la adaptación al medio al contravenir las normas de convivencia socialmente aceptadas (Gerrig y $\mathrm{Zimbardo}, 2005)$. Aunque no es necesario que todas las conductas descritas se exhiban paralelamente, resulta imprescindible que más de una de ellas se encuentre presente para adquirir significación clínica. Además, dichas conductas deben ser contextualizadas antes de calificarse como anormales (Mayorga, 2014), pues se encuentran condicionadas por la cultura que las comprende. 
En lo que respecta a la existencia de sintomatología clínica en poblaciones universitarias, las cuales están sometidas a exigencias académicas, se revela alta prevalencia de psicopatología, particularmente en lo referente a trastornos ansiosos y depresivos (65-70\%) (Campo y Cassiani, 2008; Lipson, Gaddis, Heinze, Beck, y Eisenberg, 2015). Lo que se contrapone a los índices encontrados en grupos escolares preuniversitarios, donde solo un $28 \%$ de la población adolece de síntomas y síndromes clínicamente relevantes, ligados a depresión, ideación suicida y trastornos de conducta alimentaria. Se aprecia entonces, que los valores se incrementan en las situaciones de alto riesgo, asociadas a las exigencias medioambientales estresantes, las cuales se desprenden de las demandas académicas (Casullo y Castro, 2002).

En lo que respecta a los efectos en el rendimiento académico, la sintomatología subclínica perturba los procesos intelectuales (análisis, síntesis, etc.), afectivos (motivación y actitud) y comportamentales (composición de textos, hábito de tomar notas y otros) de la/el estudiante (Naranjo, 2004), además si el hecho académico se da dentro de condiciones estresantes, la sintomatología se agudiza, produciendo efectos que imposibilitan desempeñarse adecuadamente dentro de las actividades académicas, lo cual afecta el rendimiento general del estudiantado, en especial en aquel que ya ha presentado problemas académicos previos.

Una forma técnica de demostrar el fenómeno descrito, se efectúa a través de la evaluación psicométrica, la cual detecta elementos que difícilmente se evidencian de manera directa. Diversos trabajos utilizando el Inventario de Síntomas SCL-90-R han corroborado la presencia de sintomatología clínica y subclínica en estudiantes universitarios. Estudios desarrollados sobre una población de 1277 estudiantes de la Universidad de Girona (España), han obtenido elevadas puntuaciones para los síntomas de obsesividad-compulsividad, depresión y sensibilidad interpersonal; observándose diferencias significativas entre hombres y mujeres, siendo las últimas las que presentan mayor incidencia sintomática (Caparrós, Villar, Jaume y Viñas, 2007).

Los trabajos de Gempp y Avendaño (2008), en población universitaria chilena, hallan similitudes en la sintomatología exhibida entre varones y mujeres y paralelamente propone la necesidad de confrontar en investigaciones subsiguientes las posibles diferencias entre distintos grupos de edad, a fin de determinar niveles de vulnerabilidad por edad. Una investigación comparativa ejecutada en 256 estudiantes adolescentes del País Vasco (España), Valencia (España) y Buenos Aires (Argentina), revela que mientras menor es la edad de los examinados, mayor es el nivel de malestar psicopatológico, particularmente en lo que se refiere a sensibilidad interpersonal e ideación paranoide, depresión, ansiedad, ansiedad fóbica. En cuanto a la variable género, se determina que la hostilidad y ansiedad poseen valores similares entre varones y mujeres, mientras que en los síntomas restantes (somatización, obsesiones y compulsiones, sensibilidad interpersonal, ansiedad fóbica, ideación paranoide y psicoticismo), las mujeres presentan valores mayores en todas las muestras (Casullo, Cruz, Gonzáles, y Maganto, 2003). De lo que se infiere, que la edad y el género implica una mayor vulnerabilidad y consecuente riesgo.

Ulteriormente, al analizar las dimensiones de bienestar psicológico de estudiantes adolescentes con y sin presencia de sintomatología clínica, se evidenció que un $28 \%$ de la población examinada adolecía de síntomas y síndromes de relevancia clínica, especialmente los referidos a depresión, ideación suicidas y trastornos de la conducta alimentaria (Casullo y Castro, 2002); dichos hallazgos no se hallaban asociados a la variable edad, pero si a la variable género. Un dato sumamente importante revelado en el estudio, es que estos valores se incrementan en las condiciones de alto riesgo, las cuales se desprenden de las situaciones medioambientales estresantes, entre ellas las académicas. 


\section{MÉTODo}

\section{Diseño de la investigación}

La investigación posee un diseño no experimental; de tipo cuantitativo, razón por la que utiliza encuestas y test como herramientas de producción del conocimiento (Martínez, 2011); el alcance es descriptivo y comparativo, de corte transversal, por lo que describe un fenómeno, para profundizar en su comprensión y establecer comparaciones entre las variables que lo conforman en un tiempo determinado (León, 2002).

\section{Participantes}

Los participantes del estudio fueron estudiantes de la Escuela de Psicología de la PUCESA, durante el periodo académico enero-mayo del 2014. La muestra fue de 92 estudiantes, el 77,2\% fueron mujeres y el 22,8\% hombres, con una media de edad de \# = 20.19 años; $s=2.23$. La distribución por niveles académicos fue de $41,3 \%$ en el segundo nivel, seguido por un $15,2 \%$ en tercer nivel, $10,9 \%$ en el cuarto nivel, $7,6 \%$ en el quinto nivel, 6,5\% en el octavo nivel, y 5,4\% el primero y el sexto nivel respectivamente, el resto de niveles (séptimo, noveno y décimo) se encuentra por debajo del $5 \%$ cada uno (ver Tabla 1 ).

TABLA 1

Análisis sociodemográfico

\begin{tabular}{|c|c|c|}
\hline Variables & $\begin{array}{l}\text { Estadísticos } \\
\text { Media }\end{array}$ & Desv. Est. \\
\hline \multicolumn{3}{|c|}{20,1848} \\
\hline & Frecuencia & Porcentaje \\
\hline Sexo & & \\
\hline Femenino Masculino & 7121 & $77,2 \% 22,8 \%$ \\
\hline $\begin{array}{l}\text { Condición académica } \\
\text { Sin condicionamiento Primer condicionamiento Segundo condicionamiento } \\
\text { Retirado }\end{array}$ & 30489 & $32,6 \% 52,2 \% 9,8 \% 5,4 \%$ \\
\hline $\begin{array}{l}\text { Nivel de curso } \\
\text { Primero Segundo Tercer Cuarto Quinto Sexto Séptimo Octavo Noveno Décimo }\end{array}$ & & \\
\hline & 5381410753622 & $\begin{array}{l}5,4 \% 41,3 \% 15,2 \% 10,9 \% 7,6 \% 5,4 \% 3,3 \% \\
6,5 \% 2,2 \% 2,2 \%\end{array}$ \\
\hline
\end{tabular}

Fuente: Elaboración propia

Nota: Nota: $\mathrm{n}=87$ observaciones

El tipo de muestreo empleado fue no probabilístico por conveniencia, método que "permite seleccionar aquellos casos accesibles que acepten ser incluidos" (Otzen y Manterola, 2017, p. 230). La muestra estuvo distribuida de acuerdo a la condición académica del estudiantado. Un 34,5\% estuvo constituido por un grupo de estudiantes seleccionados de manera aleatoria que no presentan situación académica en condición de vulnerabilidad (sin condicionamiento académico) y que fueron seleccionados a modo de grupo control, el 55,1\% lo conformaron estudiantes que presentan una primera situación académica de vulnerabilidad (condicionamiento académico) y el 10,4\% correspondió a estudiantes que presentan un segundo condicionamiento académico. Cabe mencionar que muestran presencia de condicionamientos académicos se encuentran concentrados mayoritariamente en el segundo y tercer nivel de formación universitaria. 


\section{Instrumentos}

Se utilizó una ficha de registro de índice promedial, para asentar de manera objetiva las calificaciones alcanzadas por el estudiantado, la existencia de condicionamiento, así como el número de condicionamientos que ostenta cada participante (Secretaría Escuela de Psicología PUCESA, 2014).

Se empleó el Inventario de los 90 Síntomas revisado de Derogatis et ál. (1976), cuestionario de los 90 síntomas revisados (SCL-90-R), en la versión del instrumento adaptada al español de Gonzáles (2002). Este instrumento se evalúa e interpreta en función de nueve dimensiones primarias: a) Somatización; b) Obsesión-compulsión; c) Sensitividad interpersonal; d) Depresión; e) Ansiedad; f) Hostilidad; g) Ansiedad fóbica; h) Ideación paranoide; e i) Psicoticismo. Además de tres índices globales de malestar psicológico: a) Índice Global de Severidad (GSI); b) Total de Síntomas Positivos (PST); y c) Índice Positivo de Malestar (PSDI). Cuenta con 90 ítems que se evalúa en una escala Likert de cinco puntos ( 0 a 4$)$ el incremento del puntaje señala mayor presencia del síntoma. El instrumento se desarrolló para evaluar sintomatología, clínica y subclínica, situación de riesgo y necesidades de atención psicológica.

Entre las propiedades psicométricas del instrumento, en la fiabilidad de las puntuaciones en la versión original de Derogatis et ál. (1976) la consistencia interna fluctúa entre .81 y .9; mientras que la estabilidad temporal $(\mathrm{r})$ se maneja en coeficientes de .78 a .9. Por otra parte, entre los ítems se encontró en población de estudiantes universitarios chilenos que los coeficientes alfa $(\alpha)$ fluctúan entre .64 en ansiedad fóbica y .82 en somatización (Gempp y Avendaño, 2008). Mientras que en estudiantes españoles de Girona dichos coeficientes fluctúan entre .69 y .97 (Caparrós et ál., 2007). Y en la población clínica argentina se encontraron puntajes de .72 a .86 y para el GSI $\alpha=.96$ (Sánchez y Ledesma, 2009).

\section{Procedimiento}

Se empleó el cuestionario de 90 síntomas SCL-90-R; el cual fue aplicado a 87 estudiantes, 57 condicionados ( 48 con primer condicionamiento y 9 con segundo condicionamiento) y 30 no condicionados, utilizados como grupo control, fueron excluidos cinco estudiantes que se retiraron durante el periodo en que se desarrolló la investigación. El proceso respetó los principios de voluntariedad y confidencialidad de cada participante, pues firmaron una carta de consentimiento informado al respecto de la información proporcionada (Mayorga, 2014).

\section{ANÁLISIS DE RESULTADOS}

En primer lugar, se incluye un análisis descriptivo de las puntuaciones obtenidas en las dimensiones primarias del SCL-90-R y de los resultados globales (GSI, PST, PSDI) que se muestran con la media (\#), la desviación estándar (s) y el percentil alcanzado en el baremo (Pe) que se toma como referencia el estudio de Gempp y Avedaño (2008), quien trabajó con población universitaria chilena. También se compara los puntajes alcanzados con el punto de corte referencias $(\mathrm{Pe}>=80)$ para conocer la presencia significativa o no de la magnitud de las puntuaciones.

Para verificar el grado de confiabilidad de la consistencia interna de las puntuaciones entre los ítems de la escala y determinar si el instrumento tuvo la capacidad de medir el constructo que se pretende cuantificar (sintomatología clínica y sub-clínica), se procedió a aplicar el coeficiente de fiabilidad Alfa de Cronbach $(\alpha)$ y conocer así el grado de fiabilidad. Consistencias equivalentes o superiores a 7 se consideran como aceptables (Kaplan y Saccuzzo, 1982; George y Mallery, 2003; Huh, DeLorme y Reid, 2006).

Para el análisis comparativo cuantitativo entre los grupos que presentan primer y segundo condicionamiento y sin condicionamiento, en el aspecto descriptivo se analizan las medias y desviaciones de 
cada uno de los grupos. Para conocer la significancia $(\mathrm{p}<.05)$ se utilizó la prueba no paramétrica de Kruskal $(\mathrm{H})$.

En cuanto al análisis categórico para conocer la situación de riesgo y la necesidad de atención psicológica en función de la severidad de la sintomatología, se utilizó las tablas normalizadas de Casullo (2004). Se establecieron tres criterios de categorización utilizando el siguiente percentil: a) No es sujeto de atención psicológica (Pe-5 al Pe-64); b), Sujeto se encuentra en situación de riesgo (Pe-65 al Pe-79); y c), El sujeto requiere atención psicológica (Pe-80 o más). Para conocer las diferencias entre las distribuciones de los grupos $(p<.05)$ se utilizó la prueba Ji Cuadrado de Pearson $(X 2)$. La gestión informática de los datos obtenidos fue analizada y procesada, a través del pr ograma SPSS en la versión 21.

\section{Resultados}

\section{Análisis descriptivo y de consistencia interna de la sintomatología psicológica}

Se observa que en el factor de Somatización los puntajes fueron de \# =1.17 puntos; $s=.829$; mientras que en el factor Obsesión-compulsión fueron \# = 1.44 puntos; $s=.758$. En el factor Sensitividad se encontró \# $=.88$ puntos; $s=.745$. Mientras que en el factor de Depresión la media obtenida corresponde a \# $=1.28 ; s$ $=.825$. En el factor Ansiedad hay valores de \# =1.03; $s=.817 ; y$ en el de Hostilidad de \# = 1 punto; $s=.834$; en Ansiedad fóbica.64 puntos; $s=.747$. Para el factor de Ideación Paranoide se presentan valores de \# = 1.02 puntos; $s=.794$ y en Psicoticismo \# = .74; $s=.703$.

El Índice de Severidad Global muestra puntuaciones de \# = 1.08 puntos; $s=.667$, mientras que el número de síntomas positivos alcanza \# = 49.45 síntomas; $s=19.211$, el Índice de Malestar Positivo fue \# = 1.84 puntos; $\mathrm{s}=.567$ (ver Tabla 2).

TABLA 2.

Análisis descriptivo de los resultados del test SC-90-R

\begin{tabular}{lccll}
\hline Indicadores & Media & Desv. & Centil & $\begin{array}{l}\text { Corte } \\
(\mathrm{P}-80)\end{array}$ \\
\hline Somatización & 1,17 &, 829 & $53-54$ & 3.70 \\
Obsesión-Compulsión & 1,44 &, 758 & $52-53$ & 3.20 \\
Sensitividad & 0,88 &, 745 & $51-52$ & 2.75 \\
Depresión & 1,28 &, 825 & $54-55$ & 3.00 \\
Ansiedad & 1,03 &, 817 & $53-54$ & 3.30 \\
Hostilidad & 1,00 &, 834 & 56 & 2.95 \\
Ansiedad fóbica & 0,64 &, 747 & 55 & 2.26 \\
Ideación Paranoide & 1,02 & 794 & 55 & 3.24 \\
Psicoticismo & 0,74 & 703 & 53 & 2.60 \\
\hline GSI & 1,08 &, 667 & $53-54$ & 2.55 \\
PST & 49,45 & 19,211 & 49 & 72 \\
PSDI & 1,84 &, 567 & $50-51$ & 3.25 \\
\hline
\end{tabular}

Fuente: Elaboración propia

Nota: Nota: $\mathrm{n}=87$ observaciones

En ninguno de los casos presentados los puntajes de las dimensiones primarias del $S C L-90-R$, así como de las escalas superan el punto de corte $(P e-80)$, para considerar que la condición global del estudiantado constituya una condición patológica específica la cual requiera intervención psicológica. Sin embargo, se debe destacar que la mayor presencia de sintomatología entre los participantes, es la relacionada con la Hostilidad (Pe-56), seguida por síntomas de Ansiedad fóbica e Ideación paranoide ( $P e-55)$, respectivamente. 
Acerca de la consistencia interna de las dimensiones primarias del $S C L-90-R$, el análisis muestra que estas fluctúan entre $\alpha=.702$ para Psicoticismo y $\alpha=.989$ para Somatización, con un análisis global de los 90 ítems de $\alpha=.947$. Estos datos apuntan a señalar que la fiabilidad de las puntuaciones entre los ítems es considerada entre moderadamente alta y alta y por lo tanto aceptable.

\section{Análisis comparativo de la severidad de la sintomatología psicológica}

En el componente de somatización se encontraron puntuaciones de \# $(N C)=1,14$ puntos; $s=.671$, \# $(1 C)$ $=1.22$ puntos; $s=.895 y \#(2 C)=1.05$ puntos; $s=1.002$; sin hallarse diferencias significativas $\mathrm{H}=.728$; p> .05. En el componente de Obsesión-compulsión fue \# $(N C)=1,29$ puntos; $s=.618$, \# $(1 C)=1.54$ puntos; $s=.803 y \#(2 C)=1.4$ puntos; $s=.931$; donde no se hallaron diferencias significativas $H=1.111 ; p>.05$. Para el componente de Sensitividad Interpersonal los resultados fueron de \# $(\mathrm{NC})=.69$ puntos; $s=.562$, \# $(1 C)=1.54$ puntos; $s=.803 y \#(2 C)=.89$ puntos; $s=.903$; con ausencia de diferencias estadísticamente significativas $H=3.818 ; p>.05$.

En la dimensión de depresión los resultados alcanzados fueron de \# $(N C)=1.08$ puntos; $s=.784$, \# $(1 C)$ $=1.37$ puntos $;=.81$ y $\#(2 C)=1.46$ puntos $; s=.995 ;$ sin encontrarse diferencias $H=2.917 ; p>.05$. Para la dimensión Ansiedad se hallaron datos de \# $(N C)=.92$ puntos; $s=.624$, \# $(1 C)=1.1$ puntos; $s=.927 y \#$ $(2 C)=1.01$ puntos; $s=.805 ;$ sin diferencias entre los grupos $H=.064 ; p>.05$ En la dimensión de Hostilidad los valores fueron de \# $(N C)=.87$ puntos; $s=.712$, \# $(1 C)=1.05$ puntos; $s=.86 y \#(2 C)=1.17$ puntos; $s$ $=1.09$; en el que no se encontraron diferencias $H=.604 ; p>.05$.

Por otra parte, en la dimensión de Ansiedad fóbica se encontraron resultados de \# $(N C)=.53$ puntos; $s$ $=.571, \#(1 C)=.74$ puntos $;=.873 y \#(2 C)=.48$ puntos $s=.452$; donde no se encontraron diferencias estadísticamente significativas $H=.445 ; p>.05$. En Ideación Paranoide se encontró puntajes de \# $(N C)=.86$ puntos; $s=.778, \#(1 C)=1.13$ puntos; $s=.815 y \#(2 C)=1.02$ puntos; $s=.724$; sin hallarse diferencias $H$ $=2.686 ; p>.05$. Mientras que en psicoticismo se encontró valores de \# $(N C)=.52$ puntos; $s=.444$, \# $(1 C)$ $=.85$ puntos; $s=.75 y \#(2 C)=.88$ puntos; $s=1.01$; en el que no se registraron diferencias significativas $H=3.615 ; p>.05$.

En el Índice global de severidad se obtuvieron resultados de \# $(N C)=.93$ puntos; $s=.54$, \# $(1 C)=1.16$ puntos; $s=.712 y \#(2 C)=1.1$ puntos; $s=.789$; en el que no se encontraron diferencias $H=1.636 ; p>.05$. Además, en el Total de Síntomas Positivos los valores fueron de \# $(N C)=47.73$ síntomas; $s=20.723$, \# $(1 C)=20.723$ síntomas; $s=17.897 \mathrm{y} \#(2 C)=49.67$ síntomas; $s=22.616$; sin hallarse diferencias entre los grupos $H=.312 ; p>.05$. Por último, en Índice de Malestar Positivo los hallazgos fueron de \# $(N C)=1.69$ puntos; $s=.476$, \# $(1 C)=1.95$ puntos $;=.5952 y \#(2 C)=1.79$ puntos; $s=.629$; sin encontrarse diferencias significativas $H=3.367 ; p>.05$ (ver Tabla 3$)$. 
TABLA 3

Descriptores de los síntomas psicológicos por categorías de condición académica

\begin{tabular}{lcllllll}
\hline Indicadores & \multicolumn{3}{c}{ SC $=30$} & \multicolumn{3}{c}{$1 \mathrm{C}=48$} & \multicolumn{2}{l}{ 2C 9} & \multirow{2}{*}{$\mathrm{H}$} \\
\cline { 2 - 6 } & Media & Desv. & Media & Desv. & Media & Desv. & \\
\hline Somatización & 1,14 &, 671 & 1,22 &, 895 & 1,05 & 1,002 & 0.728 \\
Obsesión-Compulsión & 1,29 &, 618 & 1,54 &, 803 & 1,40 & 0,931 & 1.111 \\
Sensitividad & 0,69 &, 562 & 1,00 &, 802 & 0,89 & 0,903 & 3.818 \\
Depresión & 1,08 &, 784 & 1,37 &, 810 & 1,46 & 0,995 & 2.917 \\
Ansiedad & 0,92 &, 624 & 1,10 &, 927 & 1,01 & 0,805 & 0.064 \\
Hostilidad & 0,87 &, 712 & 1,05 &, 860 & 1,17 & 1,090 & 0.604 \\
Ansiedad fóbica & 0,53 &, 571 & 0,74 &, 873 & 0,48 & 0,452 & 0.445 \\
Ideación Paranoide & 0,86 &, 778 & 1,13 &, 815 & 1,02 & 0,724 & 2.686 \\
PSicoticismo & 0,52 &, 444 & 0,85 &, 750 & 0,88 & 1,010 & 3.615 \\
\hline GSI & 0,93 &, 540 & 1,16 &, 712 & 1,10 & 0,789 & 1.632 \\
PST & 47,73 & 20,723 & 50,48 & 17,897 & 49,67 & 22,616 & 0.312 \\
PSDI & 1,69 &, 476 & 1,95 &, 595 & 1,79 & 0,629 & 3.367 \\
\hline
\end{tabular}

El conjunto de datos muestra una dinámica en la cual los puntajes del grupo de primer condicionamiento $(1 C)$ aumentan y se distancian del grupo de participantes sin condicionamientos $(S C)$, aunque a nivel general mantiene la misma distribución, lo que implica una mayor intensidad en la sintomatología psicológica. Mientras que en el grupo de segundo condicionamiento $(2 C)$, las puntuaciones bajan con respecto al grupo de $1 C$ se mantienen por encima del grupo de control $(S C)$ y la distribución varía, presentando picos en lo que corresponde a depresión y hostilidad. No se encontraron diferencias de las puntuaciones entre los grupos analizados, pero se puede estimar una tendencia a disminución o estabilización de la sintomatología psicológica, cuando la condición de vulnerabilidad se cronifica (ver Figura 1).

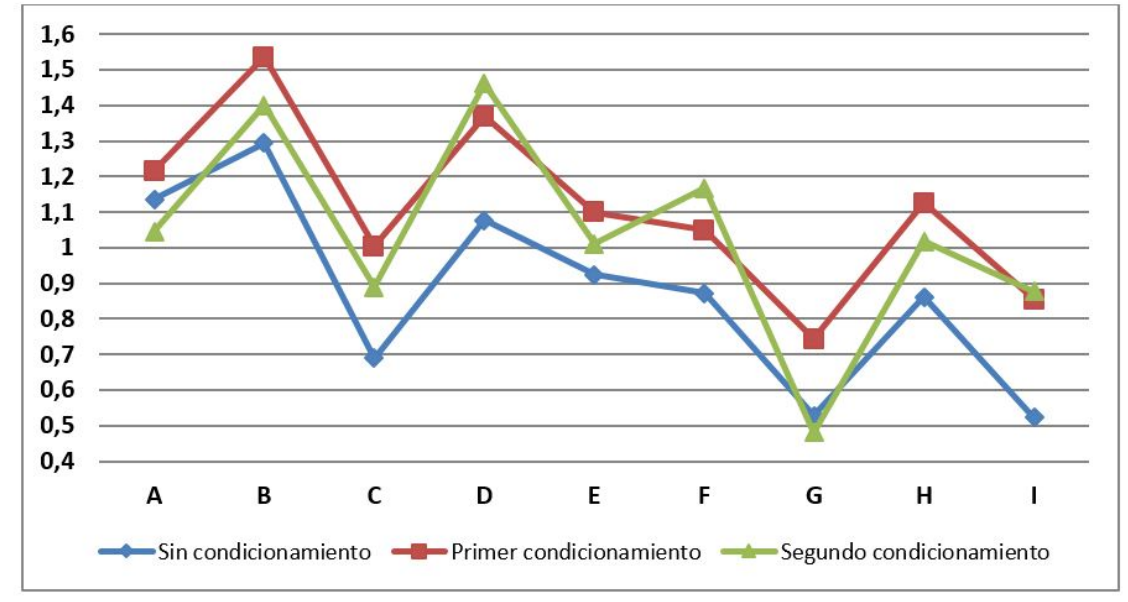

Clave: A: Somatización; B: Obsesión-Compulsión; C: Sensitividad Interpersonal; D: Depresión; E: Ansiedad; F: Hostilidad; G: Ansiedad Fóbica; H: Ideación Paranoide; I: Psicoticismo

FIGURA 1

Representación gráfica de las puntuaciones comparativas de las medias de los grupos sin condicionamiento, primer condicionamiento y segundo condicionamiento

Fuente: Elaboración propia 


\title{
Análisis de la situación de riesgo y necesidades de atención psicológicas en los participantes
}

En el grupo $S C$ y en función de la severidad de su sintomatológica el $80 \%$ no evidencia la necesidad de atención psicológica, mientras que el $20 \%$, se encuentra en una situación de riesgo a futuro. En el caso del grupo de $1 C$ en el $75 \%$ no se aprecian necesidades de atención psicológica, el 18,8\% está en situación de riesgo y el 6,2\% ya se encuentra en el segmento que requiere atención psicológica dada la severidad de su condición. Por su parte, en el grupo $2 C$, el $77,8 \%$ se encuentran en el segmento sin necesidad de atención y el $22,2 \%$ en situación de riesgo a futuro. Se anota, además que no se encontraron diferencias estadísticamente significativas en la distribución de los grupos analizados $X^{2}(4)=2.55 ; p>.05$ (ver Tabla 4).

TABLA 4

Distribuciones de las categorías diagnósticas por tipo de condición académica

\begin{tabular}{llllllll}
\hline \multirow{2}{*}{ Categorias } & \multicolumn{2}{c}{$\mathrm{SC}=30$} & \multicolumn{2}{c}{$1 \mathrm{C}=48$} & \multicolumn{2}{c}{$2 \mathrm{C}=9$} & Contraste \\
\cline { 2 - 7 } & $\mathrm{n}$ & $\%$ & $\mathrm{n}$ & $\mathrm{X}^{2}$ & $\mathrm{n}$ & $\%$ & $\mathrm{X}^{2}$ \\
\hline Sin necesidad & 24 & $80,0 \%$ & 36 & $75,0 \%$ & 7 & $77,8 \%$ & \\
En riesgo & 6 & $20,0 \%$ & 9 & $18,8 \%$ & 2 & $22,2 \%$ & $(4) 2,55$ \\
$\begin{array}{l}\text { Requiere } \\
\text { atención }\end{array}$ & 0 & $0,0 \%$ & 3 & $6,2 \%$ & 0 & $0,0 \%$ & \\
\hline
\end{tabular}

\author{
Fuente: Elaboración propia \\ Nota: Nota: ${ }^{*} \mathrm{p}<.05 ; \mathrm{X}^{2}=$ Chi cuadrado de Pearson
}

Los resultados arrojados muestran que la categoría de mayor presencia corresponde de manera global al de participantes sin necesidades de atención psicológica con un 77\% del conteo de casos. Un $19,5 \%$ se encuentran en situación de Riesgo de necesidades a futuro y un 3,5\% están en situación de requerir atención psicológica para evaluación específica y/o intervención.

\section{Discusión}

La distribución por niveles del alumnado condicionado académicamente presentó una prevalencia del 41,3\% en el segundo semestre de la carrera; seguido por un 15,2\% de estudiantes ubicados en tercer semestre y un $10,9 \%$ en cuarto nivel. Así, un 67,4\% de los condicionados se encuentran agrupados en los niveles inferiores. Se infiere que la edad del estudiantado en los primeros niveles (18-19 años aproximadamente), así como la adaptación a las exigencias de la educación superior, genera mayores niveles de vulnerabilidad en estudiantes universitarios. Estos datos son concordantes con lo mencionado por Casullo y Castro (2002).

Los resultados descriptivos globales del estudiantado evaluado determinaron que, aunque no llegan a alcanzar una intensidad clínica, los síntomas que se manifiestan en mayor medida, son los de ansiedad fóbica, hostilidad e ideación paranoide, estos se ubicaron entre los percentiles (Pe) 55 y 56 . Lo cual permite considerar que los factores estresores asociados a las exigencias académicas pueden predisponer la manifestación de síntomas psicológicos de intensidad más bien subclínica, por tratarse de una población que se encuentra dentro de límites de normalidad (Mayorga, 2014). Datos similares se encontraron en los estudios en población chilena Gempp y Avendaño (2008) y argentina y española (Casullo et ál., 2003).

Debido a que un $19,5 \%$ del estudiantado examinado de encuentra en condición de riesgo ante la manifestación de síntomas psicológicos a futuro; y dado que un 3,5\% ya se halla en situación de requerir atención psicológica como consecuencia de encontrarse con un primer condicionamiento académico. Se estima que la primera condición académica impacta a las y los estudiantes y los coloca en una situación de vulnerabilidad, pues instala en ellos sintomatología ansiosa, que tiene valor de reacción de alerta ante un 
evento traumático (Slaikeu, 1998); la cual, desde una perspectiva cognitivo-conductual determina que, al evaluar el suceso, el sujeto sienta ausencia de apoyo social y sus procesos de adaptación se vean dificultados (Fisher y Reason, 1988). Este hecho dispara los síntomas de carácter ansioso y concuerda con estudios de Osada et ál. (2010) y Del Toro et ál. (2011).

El análisis comparativo entre el grupo de $S C$ y el grupo $1 C$, muestra que la intensidad de todas las dimensiones primarias de síntomas se incrementa, aunque no de una manera suficientemente significativa para ubicarlos dentro de una población clínica. Esto implica que la percepción de peligro y riesgo que el estudiantado condicionado soporta sobre ellos, deteriora su estado de salud mental. Además, se percibe hostilidad, defensividad y suspicacia, resultados no acordes con los encontrados en otras poblaciones universitarias (Caparrós et ál., 2007; Gempp y Avendaño, 2008), en las cuales índices como el de ideación paranoide son considerablemente bajos.

Por otra parte, en el alumnado que exhibe un segundo condicionamiento se percibe una distribución sintomatológica distinta; dimensiones como depresión, hostilidad y psicoticismo se incrementan con referencia al grupo 1C. Aunque los índices de sintomatología depresiva encontrada, compaginan con los trabajos de Masi et ál. (1998), Campo y Cassiani (2008) y Lipson et ál. (2015); y los de hostilidad, también se hallan en los trabajos de Fernández y Martín (1994). Es necesario destacar, que esta actitud aparentemente paradójica de desesperanza y defensividad frente a los estudios, puede tratarse de una forma de indefensión adquirida, percepción desarrollada por el sujeto, en la de que sus esfuerzos no afectan los resultados sobre su vida cotidiana, la cual es compatible con la depresión desde un punto de vista conductual (Polaino y Vázquez, 1982). El resto de dimensiones disminuyen sus puntajes con respecto al grupo $1 C$; mientras que dimensiones como somatización y ansiedad disminuyen hasta colocarse por debajo de la media del grupo $S C$. Curiosamente, el aumento de sintomatología depresiva y la disminución de sintomatología ansiosa, podría minimizar los niveles de esfuerzo y mejor rendimiento académico.

De manera general se aprecia que un $23 \%$ de los examinados presenta riesgo y necesidad de atención, lo que también se encuentra dentro de los parámetros de normalidad pues "el riesgo de padecer cualquier trastorno mental en el último año, según criterios diagnósticos varía desde el 4,3\% hasta el 26,4\%" (Campo y Cassiani, 2008), aunque en los límites altos del espectro.

Sobre la consistencia interna de las puntuaciones en las dimensiones primarias estas fluctuaron entre 702 para psicoticismo y .989 para somatización y un global de .947 . Estos resultados apuntan a que las puntuaciones recogidas cuentan con una fiabilidad aceptable entre las respuestas de los ítems como lo proponen Kaplan y Saccuzzo (1982); George y Mallery (2003); Huh, DeLorme y Reid (2006). Además, estos datos concuerdan con los criterios de la versión original y adaptada al español (Derogatis et ál., 1976); junto con criterios en población universitaria (Gempp y Avedaño, 2008) y con población general cercana al contexto nacional (Sánchez y Ledesma, 2009). Sin embargo, estos datos se deben tomar con precaución, pues el análisis no tuvo como finalidad verificar sus propiedades psicométricas sino establecer la fiabilidad de las respuestas entre los participantes.

Los hallazgos constituyen datos relevantes para conocer la vulnerabilidad psicológica en la que el bajo rendimiento puede colocar a estudiantes universitarios y la prevalencia de ciertos síntomas psicológicos clínicos y subclínicos. Esto también permite ampliar la visión general que se tiene sobre el bajo rendimiento como un problema exclusivamente académico, sin considerar aspectos psicológicos, en especial los emocionales, que al parecer se encuentran asociados al fenómeno, tal como lo mencionan Restrepo y Malagón (1997). También se presentan implicaciones sobre los mecanismos de adaptación que se generan cuando el bajo rendimiento perdura en el tiempo en el/la estudiante.

La información puede utilizarse al respecto de los planes y estrategias que deben desarrollar los centros de educación superior sobre el seguimiento y acompañamiento psicológico al estudiantado, así como las acciones preventivas que se tomen frente al fenómeno de bajo rendimiento académico asociado a la respuesta psicológica. 


\section{LIMITACIONES}

No se contó con un análisis para conocer la presencia de diferencias significativas en segmentaciones de la población como por ejemplo el sexo o en grupos de estudiantes que posean otras condiciones académicas (por ejemplo, beneficiarios de becas), así como de otras carreras de la PUCE- Ambato. Por ello, se sugiere abordar en próximas investigaciones, la misma temática en distintos grupos de edad y en muestras equivalentes de estudiantes universitarios, a fin de establecer el grado de generalización y estabilidad de sus resultados.

Aunque los trabajos de Caparrós et ál. (2007) han obteniendo coeficientes de consistencia interna de las nueve dimensiones primarias que mide el test, que oscilan entre 0.69 y 0.97 (2007), lo que implica una alta fiabilidad y buena consistencia y validez interna. El presente estudio no contó con un análisis acerca de las propiedades psicométricas del cuestionario SCL-90-R, por lo que a futuro se requieren estudios confirmatorios acerca de la consistencia interna, la temporal y la validez tanto de convergencia, divergencia y la discriminante para poblaciones generales y específicas a nivel de estudiantes universitarios.

Finalmente, dado que los resultados de la investigación son de corte transversal, realizados durante un período académico, se estima necesario un estudio de corte longitudinal, que permita entender el fenómeno con mayor profundidad y así confirmar, enmendar o descartar los resultados presentados.

\section{REFERENCIAS}

Backhoff, E. (30 de 03 de 2016). Estudiantes de bajo rendimiento en México. El Universal. Recuperado de https:// bit.ly/2HYXIhZ

Behar, R., González, J., Ariza, M. y Aguirre, A. (2008). Trastornos de la conducta alimentaria en mujeres embarazadas controladas en atención primaria. Revista chilena de obstetricia y ginecología, 73(3), 155-162

Blanco, J. H. (2005). Fundamentos de Salud Pública. Medellín: Corporación para Investigaciones Biológicas.

Bramajo, V. y López, M. (2013). Alcoholismo en la práctica ambulatoria. (Informe No. I). Buenos Aires: Eduvirama.

Cabanach, R. G., Rodríguez, S., Valle, A., Piñeiro, I. y Millán, P. G. (2007). Metas académicas y vulnerabilidad al estrés en contextos académicos. Aula Abierta, 10, 3-16.

Campo, A. y Cassiani, C. A. (2008). Trastornos mentales más frecuentes: prevalencia y algunos factores sociodemográficos asociados. Revista Colombiana de Psiquiatría, 37(4), 559-600.

Caparrós, B., Villar, E. y Viñas, F. (2007). Symptom Check-List-90-R: fiabilidad, datos normativos y estructura factorial en estudiantes universitarios. International Journal of Clinical and Health Psychology, 7(3), 781-794.

Cano, F. J., García, J., Rodríguez, L. y Antuña, M. A. (2005). Introducción a la Psicología de la Personalidad aplicada a las Ciencias de la Educación. Sevilla: Trillas/Eduforma

Casullo, M. (2004). El Inventario de Sintomas SCL- 90-R de L. Derogatis. Buenos Aires: UBA.

Casullo, M. y Castro, A. (2002). Patrones de personalidad, síndromes clínicos. Revista de Psicopatología y Psicología Clínica, 7(2), 129-140.

Casullo, M., Cruz, M. S., Gonzáles, R. y Maganto, C. (2003). Síntomas Psicopatológicos en adolescentes: Estudio Comparativo. Revista Iberoamericana de Diagnóstico y Evaluación Psicológica, 16(2), 135-149.

Del Toro, A. Y., Gorguet, M., Pérez, Y.y Gorguet, D. A. (2011). Estrés académico en estudiantes de medicina de primer año con bajo rendimiento escolar. Medisan, 15(1), 17-22.

Derogatis, L. R., Rickels, K. y Rock, A. (1976). The SCL-90 and the MMPI: Astep in the validation of a new self-report scale. British Journal of Psychiatry, 128, 280-289.

Fernández, E. y Martín, M. (1994). Ira y hostilidad en los trastornos coronarios. Anales de Psicología, 10(2), 177-188.

Fisher, S. y Reason, J. (1988). Handbook of life stress, cognition and health. Oxford: John Wiley \& Sons.

Gempp, R. y Avendaño, C. (2008). Datos Normativos y Propiedades Psicométricas del SCL-90-R en Estudiantes Universitarios Chilenos. Terapia Psicológica, 26(1), 39-58. 
George, D. y Mallery, P. (2003). SPSS for Windows step by step: A Simple Guide and Reference. 11.0 Update. Boston: Allyn \& Bacon.

Gerrig, R. J. y Zimbardo, P. G. (2005). Psicología y Vida. México: Prentice Hall.

Gómez, C. y Rodríguez, N. (1997). Factores de Riesgo Asociados al Síndrome Depresivo en la Población Colombiana. Revista Colombiana de Psiquiatría, 26(1), 23-25.

Gonzáles, J. (2002). Cuestionario de Sintomas (SCL-90-R) de L. Derogatis. Madrid: TEA Ediciones.

Huh, J., DeLorme, D.E. y Reid, L.N. (2006). Perceived third-person effects and consumer attitudes on prevetting and banning DTC advertising. Journal of Consumer Affairs, 40(1), 90-116.

Jaude, G. (2002). Factores psicológicos que predisponen al bajo rendimiento, al fracaso y a la deserción. Valdivia: Universidad Austral de Chile.

Jiménez, Á. (2012). La eficacia de la palabra. Sobre la interpretación y los procesos de cambio subjetivo en psicoterapia. Santiago: Universidad de Chile

Kaplan, R.M. y Saccuzzo, D.P. (1982). Psychological testing: Principles, applications and issues. California: Brooks/Cole publishing.

León, J. (2002). Paradigmas de Investigación. Bucaramanga, Colombia: Universidad Santo Tomás.

Lipson, S., Gaddis, S., Heinze, J., Beck, K. y Eisenberg, D. (2015). Variations in student mental health and treatment utilization across US colleges and universities. Journal of American College Health, 63(6), 388-396. doi: http s://doi.org/10.1080/07448481.2015.1040411

Mayorga, M. (2013). Mejoramiento del rendimiento académico, a través de la modificación de los rasgos de personalidad. Un tratamiento experimental, aplicado al estudiantado con condicionamiento académico de la Escuela de Psicología de la PUCESA, durante el periodo académico. (Proyecto de investigación no publicado). Pontificia Universidad Católica del Ecuador - Sede Ambato, Ecuador.

Mayorga, M. (2014). Sintomatología Sub-clínica y el Rendimiento Académico del Estudiantado que Presenta Índice Promedial Condicionado: Caso PUCESA. (Tesis de maestría inédita). Pontificia Universidad Católica del Ecuador - Sede Ambato, Ecuador.

Masi, G., Sbrana, B., Poli, P., Tomaiuolo, F., Favilla, L. y Marcheschi, M. (1998). Depression and school functioning in non-referred adolescents: a pilot study. Child Psychiatry \& Human Development, 30(3), 161-171.

Marshall, G. y Correa, L. (2003). Clasificación de liceos de enseñanza media. Análisis de rendimiento, fracaso y vulnerabilidad. Santiago: Pontificia Universidad Católica de Chile, Departamento de Estadística.

Martínez, J. (2011). Métodos de investigación cualitativa. Silogismo, 8, 1-34.

Melillo, A. y Suárez Ojeda, E. N. (2008). Resiliencia y Educación. Buenos Aires: Paidós.

Moreira, J. F. y Telzer, E. H. (2015). Changes in family cohesion and links to depression during the college transition. Journal of Adolescents, 43, 72-82. https://doi.org/10.1016/j.adolescence.2015.05.012.

Moreta, R., López, C., Gordón, P., Ortiz, W. y Gaibor, I. (2018). Satisfacción con la vida, bienestar psicológico y social como predictores de la salud mental en ecuatorianos. Actualidades en Psicología, 32(124), 112-126

Naranjo, M. L. (2004). Enfoques conductistas, cognitivos y racional emotivos. San José, Costa Rica: Editorial de la Universidad de Costa Rica.

Núñez, F. (2001). Psicología y Salud. La Habana: Editorial Ciencias Médicas.

Osada, J., Rojas, M., Rosales, C. y Vega, J. (2010). Sintomatología ansiosa y depresiva en estudiantes de medicina. Revista de Neuro-Psiquiatría, 73(1), 15-19.

Otzen, T. y Manterola, C. (2017). Técnicas de muestreo sobre una población a estudio. Internacional Journal of Morphology, 35(1), 227-232.

Polaino, A. y Vázquez, C. (1982). La Indefensión Aprendida ¿Un modelo experimental de la depresión? Barcelona: Universidad de Barcelona, Departamento de Psiquiatría.

Paolini, E. (2005). La depresión síntoma versus depresión síndrome en el niño y el adolescente. Clínica y Análisis Grupal. Revista de Psicoterapia, Psicoanálisis y Grupo, 94(27), 55-63 
Marlon Mayorga-Lascano, et AL. Síntomas Clínicos, subclínicos y NeCesidades de atención PSicológi...

Pontificia Universidad Católica del Ecuador Sede Ambato. (2011). Reglamento General de Estudiantes. Ambato, Ecuador: PUCESA.

Restrepo, C. y Malagón, N. (1997). Factores de riesgo asociados al síndrome depresivo en la población colombiana. Revista Colombiana de Psiquiatría, 26(1), 23-25.

Rodríguez, S., Fita, S. y Torrado, M. (2004). El rendimiento académico en la transición secundaria-universidad. Revista de Educación. Temas actuales de enseñanza, 334, 391-414.

Sánchez, R. y Ledesma, R. (2009). Análisis psicométrico del inventario de síntomas revisado (SCL-90-R) en población clínica. Revista Argentina de Clínica Psicológica 18, 265-274.

Secretaría Escuela de Psicología PUCESA. (2014). Número de estudiantes condicionados. Ambato, Ecuador: PUCESA.

Slaikeu, K. A. (1998). Intervención en Crisis: Manual para prácticas e investigación. México: El Manual Moderno.

Tran, A., Tran, L., Geghre, N., Darmon, D., Rampal, M., Brandone, D. y Avillach, P. (2017). Health assessment of french university students and risk factors associated with mental health disorders. PLoS ONE, 12(11), 1-2. ht tps://doi.org/10.1371/journal.pone.0188187

Villalta, M. A. (2010). Factores de resiliencia asociados al rendimiento académico en estudiantes de contextos de alta vulnerabilidad social. Revista de Pedagogía, 31(88), 159-188.

\section{BY-NC-ND}

